\title{
Avaliação da morbidade da área doadora do retalho de Grande Dorsal
}

Evaluation of donor-site morbidity of the large dorsal retail

Evaluación de la morbilidad del área donante del colgajo dorsal ancho

Thiago Pereira de SANTANA ${ }^{1}$

Felipe de Souza SERENZA ${ }^{2}$

Fernanda Ruiz de ANDRADE ${ }^{3}$

Luis Guilherme Rosifini Alves REZENDE ${ }^{4}$

Amanda Favaro CAGNOLATI ${ }^{4}$

Alex Eduardo Calderón IRUSTA

Nilton MAZZER ${ }^{5}$

${ }^{I}$ Médico Ortopedista e Cirurgião de Mão junto ao Hospital das Clínicas da Faculdade de Medicina de Ribeirão Preto da Universidade de São Paulo (HC-FMRP-USP) 14051-140 Ribeirão Preto - SP, Brasil

${ }^{2}$ Fisioterapeuta dos Laboratórios de Análise do Movimento Humano e Avaliação Isocinética do Centro de Reabilitação do Hospital das Clínicas da Faculdade de Medicina de Ribeirão Preto da Universidade de São Paulo (HC-FMRP-USP).

Cirurgião de Mão do Hospital Estadual de Américo Brasiliense, parte do Complexo do Hospital das Clínicas da Faculdade de Medicina de Ribeirão Preto da Universidade de São Paulo (HC-FMRP-USP) 14051-140 Ribeirão Preto - SP, Brasil

${ }^{4}$ Médico Assistente da Divisão de Cirurgia da Mão, Microcirurgia e Membro Superior do Hospital das Clínicas da Faculdade de Medicina de Ribeirão Preto da Universidade de São Paulo (HC-FMRP-USP) 14051-140 Ribeirão Preto - SP, Brasil

${ }_{5}^{5}$ Professor Titular e chefe do Serviço de Cirurgia da Mão, Microcirurgia e Membro Superior do Hospital das Clínicas da Faculdade de Medicina de Ribeirão Preto da Universidade de São Paulo (HC-FMRP-USP) 14051-140 Ribeirão Preto - SP, Brasil

\section{Resumo}

Introdução: As falhas de cobertura nas extremidades são um grande desafio terapêutico. Dentre as várias opções disponíveis para manejo da cobertura encontra-se o retalho livre do Músculo Grande Dorsal, devido sua grande versatilidade e características anatômicas do pedículo. Objetivo: avaliação da morbidade da área doadora do retalho livre de grande dorsal. Métodos: 7 pacientes avaliados em relação a morbidade da área doadora, seja pelo componente estético, álgico e funcional (amplitude de movimento residual). Resultados: 71,4\% dos pacientes repetiriam o mesmo procedimento cirúrgico se necessário. 85,7\% indicariam o mesmo procedimento para outros pacientes. Discussão: Devido baixa morbidade da área doadora, o retalho livre de grande dorsal encontra-se uma excelente opção na literatura. Nossos dados corroboram com os resultados apresentados em outros estudos, porém com algumas variações na amplitude de movimento mensurada nos pacientes. Conclusões: Devido grande versatilidade e baixa morbidade na área doadora, o retalho livre de Grande Dorsal é uma excelente opção para falhas de cobertura.

Descritores: Retalhos Cirúrgicos; Microcirurgia; Procedimentos Cirúrgicos Reconstrutivos.

\section{Abstract}

Introduction: Soft tissue failure management is a major therapeutic challenge. Among the various options available for covering management is the Great Dorsal muscle free flap, due to its great versatility and anatomical characteristics of the pedicle. Objective: Evaluation of the morbidity of the donor area of the free dorsal flap. Methods: 7 patients evaluated for morbidity of the donor area, either by the aesthetic, pain and functional component (residual range of motion). Results: $71.4 \%$ of patients would repeat the same surgical procedure if necessary. $85.7 \%$ would indicate the same procedure for other patients. Discussion: Due to the low morbidity of the donor area, the free dorsal flap is an excellent option in the literature. Our data corroborate the results presented in other studies, but with some variations in the range of motion measured in patients. Conclusions: due to great versatility and low morbidity in the donor area, the Grande Dorsal free flap is an excellent option for coverage failures.

Descriptors: Surgical Flap; Microsurgery; Reconstructive Surgical Procedures.

\section{Resumen}

Introducción: No cubrir las extremidades es un gran desafío terapéutico. Entre las diversas opciones disponibles para el manejo del recubrimiento se encuentra el gran colgajo libre de músculo dorsal, debido a su gran versatilidad y características anatómicas del pedículo. Objetivo: Evaluación de la morbilidad del área donante del colgajo dorsal libre. Métodos: 7 pacientes evaluados por morbilidad del área donante, ya sea por el componente estético, dolor y funcional (rango de movimiento residual). Resultados: El 71,4\% de los pacientes repetirían el mismo procedimiento quirúrgico si fuera necesario. El 85,7\% indicaría el mismo procedimiento para otros pacientes. Discusión: Debido a la baja morbilidad del área donante, el colgajo dorsal libre es una excelente opción en la literatura. Nuestros datos corroboran los resultados presentados en otros estudios, pero con algunas variaciones en el rango de movimiento medido en pacientes. Conclusiones: Debido a su gran versatilidad y baja morbilidad en el área donante, el colgajo libre Grande Dorsal es una excelente opción para fallas de cobertura.

Descriptores: Colgajos Quirúrgicos; Microcirugía; Procedimientos Quirúrgicos Reconstructivos.

INTRODUÇÃO

As falhas de cobertura nas extremidades sempre foram um grande desafio para o Ortopedista, em especial para o Cirurgião de Mão. Sendo assim, o retalho livre do músculo Grande Dorsal se mostra bastante eficaz e versátil para cobertura de grandes lesões cutâneas por ser robusto e ter um longo pedículo $^{1}$. Conforme preferência do cirurgião o retalho pode ser muscular ou musculo-cutâneo, usado de forma livre ou rotacional preservando o pedículo íntegro.

Anatomicamente, o músculo recobre grande parte do dorso, originando-se da fáscia toracolombar, crista ilíaca e três costelas inferiores. Apresenta sua inserção na porção inferior do sulco bicipital do úmero ${ }^{2}$. Dentre suas ações biomecânicas, o Grande
Dorsal é responsável pela rotação medial do úmero, adução e extensão do ombro, rebaixamento do braço elevado e rotação descendente da escápula ${ }^{3}$. Em teoria sua retirada como retalho livre gera uma disfunção do ombro, apesar de trabalhos defenderem mínima incapacidade funcional a longo prazo ${ }^{4}$.

Benditte-Klepetko et al. comparando diversos tipos de retalhos demonstraram uma taxa de insatisfação de $10 \%$ após retalho de Grande Dorsal. As complicações documentadas incluíram 3\% de necrose parcial, $12 \%$ de infecção, $3 \%$ de hematoma e $6 \%$ de revisões ${ }^{5}$.

Dentre as complicações, a formação de seroma é a mais comum, atingindo valores tão altos quanto $62 \%^{6-13}$. Outra complicação descrita é a 
formação de tumor desmóide na área doadora ${ }^{14}$. O objetivo deste trabalho é avaliar a morbidade na área doadora após retalho livre musculocutâneo de Grande Dorsal.

\section{MATERIAL E MÉTODO}

Trata-se de um estudo transversal, através de aplicação de questionário e avaliação clínicofuncional dos pacientes. Foi realizada pesquisa de prontuário entre os anos 2014 a 2016 para identificação de todos os pacientes operados no serviço, submetidos a retalho de Grande Dorsal. O período mínimo de seguimento pós-operatório é de 12 meses.

Os critérios de exclusão foram pacientes menores de 18 anos de idade, área receptora no membro superior e todos aqueles com outras patologias agudas ou crônicas no membro a ser estudado que pudessem interferir nos resultados. A avaliação foi realizada durante o seguimento pósoperatório pelo protocolo seguindo 12, 18 e 24 meses do período pós-operatório. O questionário DASH (Disabilities of the Arm, Shoulder and Hand) foi aplicado para avaliação da função global do membro. Os pacientes também responderam à avaliação subjetiva conforme apresentado na Tabela 1. O paciente também respondeu a mais duas perguntas com resposta "SIM" ou "NÃO" (Tabela 2).

Tabela 1. Avaliação subjetiva do paciente

Questões

doadora

Satisfação estética com área doadora

Percepção funcional do ombro

Fonte: Dados da Pesquisa

Tabela 2. Avaliação objetiva do paciente

Questões Escala numérica de o a 10

Você escolheria este mesmo método de

cobertura caso necessitasse novamente?

Você indicaria este método de cobertura para

outros pacientes?

Fonte: Dados da Pesquisa

\section{A avaliação do arco de movimento e força do} ombro foi realizado considerando o membro contralateral não operado como comparação. $\mathrm{O}$ ombro de cada paciente foi avaliado nos seguintes movimentos: flexão, extensão, abdução, adução, rotação externa e interna. $\mathrm{O}$ arco de movimento foi medido com goniômetro e a força através do dinamômetro isométrico Lafayette instrument (Lafayette ${ }^{\circledR}$, Lafayette Instrument Company, Ind., EUA), cujas medidas são geradas na unidade quilograma força (Kgf). A escolha do instrumento se deve à alta aceitação na literatura e fácil reprodutibilidade entre os trabalhos ${ }^{15-17}$. Os pacientes foram orientados a usar o aparelho e realizado todos os testes antes da mensuração final. As medidas utilizadas no trabalho foram obtidas após repouso de pelo menos 5 minutos, por meio de exercícios isométricos (Tabela 3). A análise estatística foi feita utilizando o software IBM SPSS ${ }^{\circ}$, com ênfase ao índice de Correlação de Pearson e ao valor de significância. $O$ primeiro, gerando valores de 1 (menos um) até +1 (um positivo), demonstraria correlação direta entre as variáveis frente a valores positivos, e correlação inversa para valores negativos. Quanto mais afastado do valor 0(zero) melhor a correlação. O valor de significância foi considerado importante se menor que 0,05 .

\begin{tabular}{|c|c|}
\hline Exercício Isométrico & Parâmetros \\
\hline Flexão & $\begin{array}{l}\text { Posição supina, ombro em flexão e abdução } \\
\text { neutra, cotovelo levemente flexionado, } \\
\text { antebraço em pronação. Dinamômetro } \\
\text { posicionado no úmero lateral, supra-condilar. }\end{array}$ \\
\hline Extensão & \begin{tabular}{ll}
\multicolumn{3}{l}{ Posição sentada, ombro e cotovelo em neutro, } \\
antebraço pronado. & Dinamômetro \\
posicionado no olecrano &
\end{tabular} \\
\hline Abdução & $\begin{array}{l}\text { Posição sentada, ombro em } 90^{\circ} \text { de abdução, } \\
\text { cotovelo levemente flexionado, antebraço em } \\
\text { pronação. Dinamômetro posicionado no } \\
\text { úmero lateral, supra-condilar }\end{array}$ \\
\hline Adução & $\begin{array}{l}\text { Posição sentada, ombro em } 30^{\circ} \text { de abdução, } \\
\text { cotovelo levemente flexionado, antebraço em } \\
\text { pronação. Dinamômetro posicionado no } \\
\text { úmero medial, supra-condilar }\end{array}$ \\
\hline Rotação Externa & $\begin{array}{l}\text { Posição sentada, ombro em } 45^{\circ} \text { de flexão, } \\
\text { cotovelo em } 135^{\circ} \text { de flexão. Dinamômetro } \\
\text { posicionado no estiloide da ulna }\end{array}$ \\
\hline Rotação Interna & $\begin{array}{l}\text { Posição sentada, ombro em } 45^{\circ} \text { de flexão, } \\
\text { cotovelo em } 135^{\circ} \text { de flexão. Dinamômetro } \\
\text { posicionado no estiloide da ulna. }\end{array}$ \\
\hline
\end{tabular}

RESULTADOS

Por meio do acesso ao banco de dados do hospital foram identificados 17 pacientes submetidos à retalho de Grande Dorsal no período entre $2014 \mathrm{e}$ 2016. Quatro destes pacientes eram menores de idade (4,5,6 e 10 anos), três tiveram retalho realizado para cobertura de falha em membro superior, um paciente havia se mudado de cidade e dois pacientes não foram contatados pois não atenderam ao telefonema. Todos esses pacientes foram excluídos do trabalho, portanto avaliamos no total 7 pacientes. A idade variou de 24 a 68, com média de 46 anos. Total de 5 homens e 2 mulheres. Quatro retalhos realizados do lado direito e 3 do lado esquerdo. Seis pacientes necessitaram de cobertura após trauma (sendo um submetido à artrodese tíbio-társica primária) e um para cobertura após ressecção de melanoma na perna. Um paciente apresentou necrose completa do retalho, sendo subsequentemente tratado com curativo a vácuo e enxertia. Não houve relato de seroma que necessitasse de tratamento cirúrgico entre esses pacientes.

Pela resposta aos questionários $5 / 7(71,4 \%)$ optariam pelo mesmo tipo de cobertura caso necessário e 6/7 (85,7\%) indicariam o mesmo procedimento para outros pacientes. A função do ombro e satisfação com o procedimento de forma geral foram bastante altos (Tabela 4). Apenas um paciente (número 7) referiu limitação específica, nos exercícios de Puxada Vertical realizados em Academia, nos quais se faz necessário força de adução dos ombros e rebaixamento dos braços após elevação. 
Tabela 4. Função do Ombro e Resultado Funcional

\begin{tabular}{l|l|l|l}
\hline & DASH & $\begin{array}{l}\text { Dor no ombro após } \\
\text { procedimento* }\end{array}$ & $\begin{array}{l}\text { Função do ombro } \\
\text { após procedimento* }\end{array}$ \\
\hline Paciente 1 & 3,33 & 0 & 10 \\
\hline Paciente 2 & 5 & 0 & 10 \\
\hline Paciente 3 & 0 & 0 & 10 \\
\hline Paciente 4 & 2,5 & 2 & 8 \\
\hline Paciente 5 & 0,83 & 0 & 10 \\
\hline Paciente 6 & O & o & 10 \\
\hline Paciente 7 & O & o & 10 \\
\hline MÉ DIA & 1,66 & 0,28 & 9,71 \\
\hline Fonte: Dados da Pesquisa &
\end{tabular}

Durante as entrevistas observamos que a principal queixa dos pacientes era com o aspecto estético da cicatriz. Uma paciente ficou completamente insatisfeita dando nota 0 (zero) à cicatriz. A avaliação objetiva e subjetiva foram compatíveis entre si (Tabela 5), sendo o paciente número 5 com a melhor avaliação estética (Figura 1).

Tabela 5. Avaliação estética da cicatriz

\begin{tabular}{|c|c|c|c|c|}
\hline & $\begin{array}{l}\text { Satisfação estética } \\
\text { subjetiva* }\end{array}$ & $\begin{array}{l}\text { POSAS } \\
\text { paciente }\end{array}$ & $\begin{array}{l}\text { POSAS } \\
\text { observador }\end{array}$ & Vancouver \\
\hline Paciente 1 & 5 & 29 & 27 & 2 \\
\hline Paciente 2 & 10 & 15 & 22 & 1 \\
\hline Paciente 3 & 5 & 39 & 28 & 6 \\
\hline Paciente 4 & 0 & 50 & 32 & 5 \\
\hline Paciente 5 & 10 & 15 & 18 & 1 \\
\hline Paciente 6 & 10 & 27 & 19 & 2 \\
\hline Paciente 7 & 7 & 18 & 11 & 1 \\
\hline MÉDIA & 6,71 & 27,57 & 22,42 & 2,57 \\
\hline
\end{tabular}

Fonte: Dados da Pesquisa

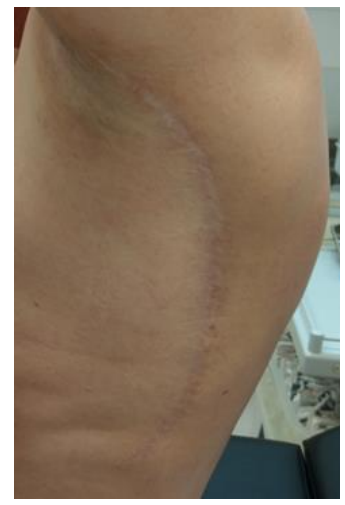

Figura 1: Aspecto estético da área doadora (Fonte: Dados da Pesquisa)

Ao se avaliar o arco de movimento encontramos diferenças mínimas em todos os itens avaliados: flexão, extensão, abdução, adução, rotação interna e rotação externa (Figuras 2 e 3 ). A maior diferença foi de $3,7^{\circ}$ na flexão ativa lado operado $\left(168,57^{\circ}\right)$ em ao contralateral $\left(164,85^{\circ}\right)$. Este valor também foi observado na extensão passiva e abdução ativa.

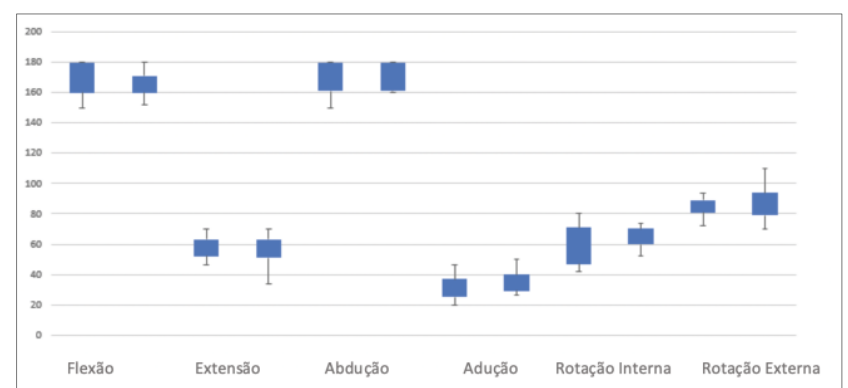

Figura 2: Amplitude de movimento ativo. Lado operado (esquerda) e Lado Controle (direita) (Fonte: Dados da Pesquisa).

Por fim, apesar de ser pouco perceptível pelo paciente houve diferença de força entre o lado operado e o lado não operado em todos os movimentos com forte correlação estatística. A força menos afetada foi de Flexão e as principais diferenças ocorreram para Extensão, Abdução e Adução (Tabela 6).

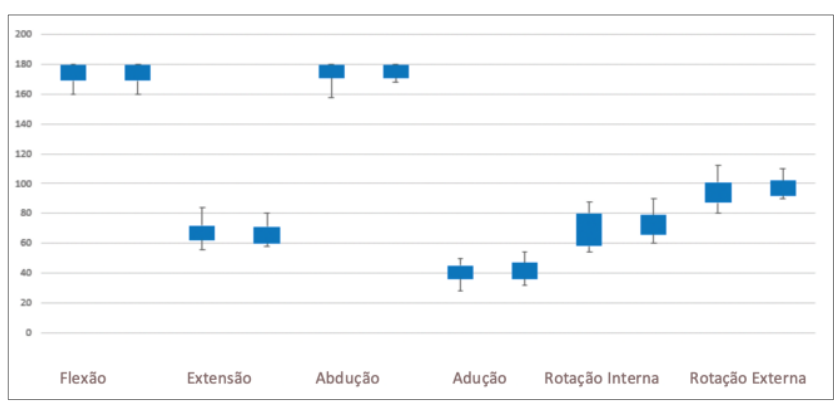

Figura 3: Amplitude de movimento passivo. Lado operado (esquerda) e Lado Controle (direita) (Fonte: Dados da Pesquisa).

\begin{tabular}{|c|c|c|c|c|}
\hline & & $\begin{array}{l}\text { Valor } \\
\text { Absoluto } \\
\text { (Kgf) }\end{array}$ & $\begin{array}{l}\text { Correlação } \\
\text { de Pearson }\end{array}$ & Significância \\
\hline Flexão & $\begin{array}{l}\text { Lado Operado } \\
\text { Controle }\end{array}$ & $\begin{array}{l}15,31 \\
16,70\end{array}$ & 0,764 & 0,046 \\
\hline Extensão & $\begin{array}{l}\text { Lado Operado } \\
\text { Controle }\end{array}$ & $\begin{array}{l}16,74 \\
18,59\end{array}$ & 0,970 & $<0,001$ \\
\hline Abdução & $\begin{array}{l}\text { Lado Operado } \\
\text { Controle }\end{array}$ & $\begin{array}{l}6,92 \\
7,50\end{array}$ & 0,974 & $<0,001$ \\
\hline Adução & $\begin{array}{l}\text { Lado Operado } \\
\text { Controle }\end{array}$ & $\begin{array}{l}9,14 \\
9,96\end{array}$ & 0,990 & $<0,001$ \\
\hline $\begin{array}{l}\text { Rotação } \\
\text { Interna }\end{array}$ & $\begin{array}{l}\text { Lado Operado } \\
\text { Controle }\end{array}$ & $\begin{array}{l}13,63 \\
14,30\end{array}$ & 0,886 & 0,008 \\
\hline $\begin{array}{l}\text { Rotação } \\
\text { Externa }\end{array}$ & $\begin{array}{l}\text { Lado Operado } \\
\text { Controle }\end{array}$ & $\begin{array}{l}10,34 \\
10,15\end{array}$ & 0,935 & 0,002 \\
\hline
\end{tabular}

\section{DISCUSSÃO}

Os dados obtidos nesse trabalho corroboram com muitos relatos da literatura ${ }^{3,18}$, nos quais é defendida que a retirada do músculo Grande Dorsal não gera queixas funcionais por parte do paciente. Apesar disto detectamos redução da força em todos os movimentos do ombro apesar de afetar minimamente a arco de movimento.

A avaliação das tabelas de arco de movimento permite observar que alguns movimentos tais como adução, rotação interna e rotação externa, tanto ativos como passivos sofreram leve aumento com relação ao lado não operado. A diferença no valor absoluto foi muito baixa, sem significância estatística. Porém esse fato pode ser justificado pela perda do tônus do músculo grande dorsal que poderia limitar levemente o arco de movimento. Esses resultados vão ao encontro daqueles verificados na literatura que apontam os movimentos de flexão e abdução como os mais afetados ${ }^{19}$.

O esperado teoricamente após realização do retalho de Grande Dorsal seria perda de força principalmente para extensão e em menor quantidade para adução e rotação externa ${ }^{20}$. No nosso trabalho, com exceção da flexão, todos os demais movimentos apresentaram perda significativa de força. Acreditamos que a ausência do músculo Grande Dorsal possa provocar alteração da biomecânica de toda cintura escapular e do movimento escapulotorácico. 
Mesmo com esses dados objetivos, de forma geral os pacientes não relatam alteração funcional no ombro para as atividades do dia-a-dia. Isso é comprovado pelo valor do questionário DASH com média de 1,66. Na avaliação subjetiva sobre a função do ombro 6 em 7 deram nota 10. Apenas uma paciente deu nota 8 , sendo a única a relatar dor no ombro no momento da avaliação. Portanto essa perda funcional pode estar mais relacionada à dor do que à perda propriamente dita da função do Grande Dorsal.

No outro extremo estão as queixas estéticas, unânime para os pacientes. O microcirurgião deve levar em consideração esse desconforto ao decidir qual tipo de cobertura realizar. Altos níveis de insatisfação foram observados em nosso estudo. Nossa baixa incidência de seroma pós-operatório pode dever-se ao uso de sutura do tipo quilting e ao uso de dreno à vácuo em todos os pacientes por pelo menos 48 horas.

Importante ressalvar que o presente estudo contou com um número pequeno de pacientes e o ombro contralateral foi utilizado como controle, o que explicaria a diferenças nas medidas por si só. Tais observações nos levam a uma proposta ideal de avaliação na qual, a avaliação pré-operatória inicial seria seguida por outra após um ano da cirurgia no mesmo membro.

Também acreditamos que a retirada do Grande Dorsal, mesmo levando à perda de força em tornos de alguns quilogramas-força nos movimentos do ombro, pode ser significativa para atletas de alto rendimento e profissionais do esporte. Nenhum de nossos pacientes encaixava-se nesse perfil e esse pode ser tema para novos estudos.

\section{CONCLUSÃO}

Concluímos que o retalho de Grande Dorsal para cobertura de falhas cutâneas ainda é uma excelente opção para o cirurgião por sua anatomia constante e versatilidade. A preocupação com a função do ombro não deve ser fator impeditivo para a realização da cirurgia, porém o paciente deve ser informado do aspecto estético negativo do procedimento e a decisão tomada em conjunto com o mesmo.

\section{REFERÊNCIAS}

1. Pederson WC. Nonmicrosurgical coverage of the upper extremity. In: Green's Operative Hand Surgery. Wolfe SW, Hotchkiss RN, Pederson WC et al. (Org.). 7. Ed. Philadelphia: Elsevier, 2017. cap. 44, p. 1528-1573. v. 2.

2. Jobe MT. Microcirurgia. In: Cirurgia Ortopédica de Campbell. Canale ST (Org.). 10. ed. [S.1.]: São Paulo: Manole, 2006. cap. 60, p. 3287-3371. v. 4.

3. Spear SL, Hess CL. A review of the biomechanical and functional changes in the shoulder following transfer of the latissimus dorsi muscles. Plast Reconstr Surg. 2005;115(7):2070-73.
4. Paolini G, Amoroso M, Pugliese P, Longo B, Santanelli F. Functional sequelae following bilateral mastectomy and immediate reconstruction with latissimus dorsi flap: mediumterm follow-up. J Plast Surg Hand Surg. 2014; 48(2):99-103.

5. Benditte-Klepetko HC, Lutgendorff F, Kästenbauer T, Deutinger M, van der Horst CM. Analysis of patient satisfaction and donor-site morbidity after different types of breast reconstruction. Scand J Surg. 2014;103(4):249-55.

6. Nagarkar P, Lakhiani C, Cheng A, Lee M, Teotia S, Saint-Cyr M. No-drain DIEP Flap Donor-site Closure Using Barbed Progressive Tension Sutures. Plast Reconstr Surg Glob Open. 2016;4(4):e672.

7. Mannu GS, Qurihi K, Carey F, Ahmad MA, Hussien M. Quilting after mastectomy significantly reduces seroma formation. S Afr $\mathrm{j}$ surg. 2015;53(2):50-4.

8. Sajid MS, Betal D, Akhter N, Rapisarda IF, Bonomi R. Prevention of postoperative seromarelated morbidity by quilting of latissimus dorsi flap donor site: a systematic review. Clin Breast Cancer. 2011;11(6):357-63.

9. Lee KT, Mun GH. Fibrin Sealants and Quilting Suture for Prevention of Seroma Formation Following Latissimus Dorsi Muscle Harvest: A Systematic Review and Meta-analysis. Aesthetic Plast Surg. 2015;39(3):399-409.

10. Llewellyn-Bennett R, Greenwood R, Benson JR, English R, Turner J, Rayter Z et al. Randomized clinical trial on the effect of fibrin sealant on latissimus dorsi donor-site seroma formation after breast reconstruction. Br J Surg. 2012; 99(10):1381-1388.

11. Cheng HT, Hsu YC, Wu CI. Quilting sutures, fibrin tissue adhesive or both in reducing the incidence of seroma in the latissimus dorsi flap donor site? An evidence-based analysis. J Plast Reconstr Aesthet Surg. 2014;67(6):881-82.

12. Lee J, Bae Y, Jung JH, Kim WW, Hwang SO, Kwon TJ et al. Effects of Quilting Suture Interval on Donor Site Seromas After Breast Reconstruction With Latissimus Dorsi Muscle Flap: A Randomized Trial. Clin Breast Cancer. 2016;16(6):e159-64.

13. Watanabe K, Morihisa Y, Gotanda K, Kiyokawa $\mathrm{K}$. A case of a chronic expanding hematoma that developed in a latissimus dorsi muscle flap donor site. J Plast Reconstr Aesthet Surg. 2013; 66(6):872-74.

14. Pyon JK, Kang BY, Mun GH, Bang SI, Oh KS, Lim SY. Extra-Abdominal Desmoid Tumor in the Donor Site of an Extended Latissimus Dorsi Flap. Arch Plast Surg. 2016;43(1):114-16.

15. Cools AM, De Wilde L, Van Tongel A, Ceyssens 
C, Ryckewaert R, Cambier DC. Measuring shoulder external and internal rotation strength and range of motion: comprehensive intra-rater and inter-rater reliability study of several testing protocols. J Shoulder Elbow Surg. 2014; 23(10):1454-61.

16. Katoh M. Test-retest reliability of isometric shoulder muscle strength measurement with a handheld dynamometer and belt. J Phys Ther Sci. 2015;27(6):1719-22.

17. Celik D, Dirican A, Baltaci G. Intrarater reliability of assessing strength of the shoulder and scapular muscles. J Sport Rehabil. 2012;21(1):1-5.

18. Button J, Scott J, Taghizadeh R, Weiler-Mithoff E, Hart AM. Shoulder function following autologous latissimus dorsi breast reconstruction. A prospective three year observational study comparing quilting and non-quilting donor site techniques. J Plast Reconstr Aesthet Surg. 2010;63(9):1505-12.

19. Lee KT, Mun GH. A systematic review of functional donor-site morbidity after latissimus dorsi muscle transfer. Plast Reconstr Surg. 2014;134(2):303-14.

20. Garusi C, Manconi A, Lanni G, et al. Shoulder function after breast reconstruction with the latissimus dorsi flap: A prospective cohort study Combining DASH score and objective evaluation. Breast. 2016;27:78-86.

\section{CONFLITO DE INTERESSES}

Os autores declaram não haver conflitos de interesse.

\section{AUTOR PARA CORRESPONDÊNCIA}

\section{Luis Guilherme Rosifini Alves Rezende}

lgrezende@usp.br
Submetido em 31/12/2019

Aceito em 11/01/2020 